\title{
Automatic Generation of Building Temperature Maps from Hyperspectral Data
}

\begin{abstract}
A method to automatically retrieve Building Surface Temperature maps using hyperspectral imagery is presented. The approach can be conceptually described by considering two different problems: the first consists in the design of an automatic procedure for the extraction of building surfaces from the hyperspectral image. Such an issue has been addressed using both unsupervised and supervised neural networks. The second problem deals with the retrieval of Land Surface Temperature (LST) from the same image. The final step is the merging of the temperature map with the buildings mask. It is worthwhile to observe that the proposed approach aims at retrieving the temperature values by reducing manual editing and the use of ancillary data to minimum level. The obtained results show an accuracy in building identification of 83.7 \% and a RMSE (Root Mean Square Error) in temperature retrieval of 1.59 K. The importance of this methodology has to be considered within the studies on Urban Heat Islands, which is becoming an important issue in Urban Management politics.
\end{abstract}

